\title{
A Singular Initial-Value Problem for Second-Order Differential Equations
}

\begin{abstract}
Afgan Aslanov
Mathematics and Computing Department, Beykent University, Ayazağa, Şişli, 34396 Istanbul, Turkey

Correspondence should be addressed to Afgan Aslanov; afganaslanov@beykent.edu.tr

Received 4 December 2013; Accepted 10 March 2014; Published 9 April 2014

Academic Editor: Elena Braverman

Copyright (c) 2014 Afgan Aslanov. This is an open access article distributed under the Creative Commons Attribution License, which permits unrestricted use, distribution, and reproduction in any medium, provided the original work is properly cited.

We are interested in the existence of solutions to initial-value problems for second-order nonlinear singular differential equations. We show that the existence of a solution can be explained in terms of a more simple initial-value problem. Local existence and uniqueness of solutions are proven under conditions which are considerably weaker than previously known conditions.
\end{abstract}

\section{Introduction}

In recent years, the studies of singular initial-value problems (IVPs) of the type

$$
x^{\prime \prime}+2 t^{-1} x^{\prime}+x^{n}(t)=0, \quad x(0)=1, x^{\prime}(0)=0,
$$

have attracted the attention of many mathematicians and physicists (see, e.g., [1-8]). It is the aim of this paper to study the more general IVPs of the form

$$
\begin{array}{r}
x^{\prime \prime}+p(t) x^{\prime}+q(t, x(t))=0, \quad x(0)=a, x^{\prime}(0)=b, \\
t>0,
\end{array}
$$

and to make further progress beyond the achievements made so far in this regard. The case $q=f(t) g(x)$ corresponds to Emden-Fowler equations [3, 8-10].

The function $p(t)$ in (2) may be singular at $t=0$. Note that the problem (2) extends some well-known IVPs in the literature; see, for example, [11-18].

In the case $b=0$ the existence of the solution for the problem (2) has been studied in [19], where the authors demonstrated the importance of the condition $b=0$ for the existence. We find the conditions for $p(t)$ and $q(t, x(t))$ to guarantee the existence of the solution for $b \neq 0$.

\section{Existence Theorems}

We say that $x(t)$ is a solution to (2) if and only if there exists some $T>0$ such that

(1) $x(t)$ and $x^{\prime}(t)$ are absolutely continuous on $[0, T]$,

(2) $x(t)$ satisfies the equation given in (2) a.e. on $[0, T]$,

(3) $x(t)$ satisfies the initial conditions given in (2).

In this section, we generalize the existence theorem of solutions in [19] (see also, [20]).

Theorem 1. Let $p$ and $q$ satisfy the following conditions:

(D1) $p$ is measurable on $[0,1]$;

(D2) $p \geq 0$;

(D3) $\int_{0}^{1} s p(s) d s<\infty$;

(D4) there exist $\alpha, \beta$ with $\alpha<a<\beta$ and $K>0$ such that

(a) for each $t \in(0,1], q(t, \cdot)$ is continuous on $[\alpha, \beta]$;

(b) for each $x \in[\alpha, \beta], q(\cdot, x)$ is measurable on $[0,1]$;

(c) $|q(t, x)| \leq K$.

Then a solution to the initial-value problem (2) with $b=0$ exists.

In [5] the authors demonstrated the importance of the condition $b=0$ for the existence. 
To overcome the difficulties in the case $b \neq 0$ we consider a generalization of Theorem 1 and show that the statement of the theorem is true without condition (D3) and with weaker conditions on $q(t, x)$.

Theorem 2. Suppose that $p(t)$ is integrable on the interval $[c, d]$ for all $c>0$ and $p$ and $q$ satisfy the following conditions:

(D1) $p$ is measurable on $[0,1]$;

(D2) $p \geq 0$;

(D4 $\left.{ }^{*}\right)$ there exist $\alpha, \beta$ with $\alpha<a<\beta, K>0$, and an integrable (improper, in general) $\varphi(t)$ such that

(a) for each $t \in(0,1], q(t, \cdot)$ is continuous on $[\alpha, \beta]$;

(b) for each $x \in[\alpha, \beta], q(\cdot, x)$ is measurable on $[0,1]$;

(c) $|q(t, x)-\varphi(t)| \leq K$.

Then a solution to the initial-value problem (2) exists for all $b \in R$ such that

$$
b=z^{\prime}(0)
$$

where $z(t) \in C[0,1]$ is a solution of the problem

$$
z^{\prime \prime}+p(t) z^{\prime}+\varphi(t)=0, \quad z(0)=a, z^{\prime}(0)=b, t>0 .
$$

That is we suppose the existence of solution of the problem (4) for some $\varphi(t)$. For the problems with $b=0$, the initialvalue problem (4) always has a solution $z(t)=a$, for $\varphi(t) \equiv 0$. So Theorem 1 corresponds to the cases $\varphi(t)=0$ and $z(t)=a$.

One of the advantages of Theorem 2 is that the problem (4) always has a solution for some appropriate $\varphi(t)$; for example, for $\varphi(t)=-b p(t)$, the problem (4) has a solution $z(t)=a+b t$. The conclusion of the theorem remains valid for all solutions of (4).

It is also clear from the conclusion of Theorem 2 that the interval $[0,1]$ can be taken as $\left[0, t_{0}\right]$ for some small enough $t_{0}>0$.

Proof of Theorem 2. For $t \in(0,1]$, we define the functions

$$
\begin{aligned}
& h(t) \equiv \exp \left(\int_{1}^{t} p(s) d s\right) \geq 0, \\
& h_{1}(t)=\exp \left(-\int_{1}^{t} p(s) d s\right), \\
& E(t)=\int_{1}^{t} h_{1}(s) d s .
\end{aligned}
$$

The function $h(t)$ is a bounded function which is continuous for $t \in(0,1]$. It is continuous or has a removable discontinuity at $t=0$ and is differentiable a.e.
We will show that the problem (2) is equivalent to the following integral equation:

$$
\begin{aligned}
x(t)=\int_{0}^{t} & \left(E(s) e^{\int_{1}^{s} p(\tau) d \tau}-E(t) e^{\int_{1}^{s} p(\tau) d \tau}\right) \\
& \times[q(s, x(s))-\varphi(s)] d s+z(t) .
\end{aligned}
$$

First, let us show the existence of the integral in (6). We have for any $\delta>0$ that

$$
\begin{aligned}
& \left|\int_{\delta}^{t} E(s) e^{\int_{1}^{s} p(\tau) d \tau}[q(s, x(s))-\varphi(s)] d s\right| \\
& \leq K\left|\int_{\delta}^{t} E(s) e^{\int_{1}^{s} p(\tau) d \tau} d s\right| \\
& =K\left|\int_{\delta}^{t} \int_{1}^{s} h_{1}(u) e^{\int_{1}^{s} p(\tau) d \tau} d u d s\right| \\
& =K\left|\int_{\delta}^{t} \int_{1}^{s} e^{-\int_{1}^{u} p(v) d v} e^{\int_{1}^{s} p(\tau) d \tau} d u d s\right| .
\end{aligned}
$$

It follows from $u \geq s$ on the set $[s, 1] \times[0, t]$ that

$$
\begin{gathered}
e^{-\int_{1}^{u} p(v) d v} e^{\int_{1}^{s} p(\tau) d \tau}=e^{-\int_{s}^{u} p(v) d v} \leq 1 \\
\left|\int_{\delta}^{t} E(s) e^{\int_{1}^{s} p(\tau) d \tau}[q(s, x(s))-\varphi(s)] d s\right| \leq K\left|t-\frac{t^{2}}{2}\right| .
\end{gathered}
$$

In like manner we obtain

$$
\begin{aligned}
& \left|\int_{\delta}^{t} E(t) e^{\int_{1}^{s} p(\tau) d \tau}[q(s, x(s))-\varphi(s)] d s\right| \\
& \quad \leq K\left|\int_{\delta}^{t} E(t) e^{\int_{1}^{s} p(\tau) d \tau} d s\right|=K\left|\int_{\delta}^{t} \int_{1}^{t} h_{1}(u) e^{\int_{1}^{s} p(\tau) d \tau} d u d s\right| \\
& \quad \leq K\left|t-t^{2}\right| .
\end{aligned}
$$

So the right-hand side of (6) makes sense for any $p(t) \geq 0$ and $|q(t, x(t))-\varphi(t)| \leq K$ and

$$
\begin{aligned}
\lim _{\delta \rightarrow 0} \int_{\delta}^{t}\left(E(s) e^{\int_{1}^{s} p(\tau) d \tau}-E(t) e^{\int_{1}^{s} p(\tau) d \tau}\right) \\
\times[q(s, x(s))-\varphi(s)] d s+z(t) \\
=\int_{0}^{t}\left(E(s) e^{\int_{1}^{s} p(\tau) d \tau}-E(t) e^{\int_{1}^{s} p(\tau) d \tau}\right) \\
\times[q(s, x(s))-\varphi(s)] d s+z(t) .
\end{aligned}
$$


Now let us calculate the derivatives $x^{\prime}(t)$ and $x^{\prime \prime}(t)$ from (6) by using the Leibniz rule:

$$
\begin{aligned}
x^{\prime}(t)= & \left(\int_{0}^{t} E(s) e^{\int_{1}^{s} p(\tau) d \tau}[q(s, x(s))-\varphi(s)] d s\right. \\
& \left.-\int_{0}^{t} E(t) e^{\int_{1}^{s} p(\tau) d \tau}[q(s, x(s))-\varphi(s)] d s+z(t)\right)^{\prime} \\
= & E(t) e^{\int_{1}^{t} p(\tau) d \tau}[q(t, x(t))-\varphi(t)] \\
& -E^{\prime}(t) \int_{0}^{t} e^{\int_{1}^{s} p(\tau) d \tau}[q(s, x(s))-\varphi(s)] d s \\
& -E(t) e^{\int_{1}^{t} p(\tau) d \tau}[q(t, x(t))-\varphi(t)]+z^{\prime}(t) \\
= & -h_{1}(t) \int_{0}^{t} e^{\int_{1}^{s} p(\tau) d \tau}[q(s, x(s))-\varphi(s)] d s+z^{\prime}(t), \\
x^{\prime \prime}(t)= & \left.-h_{1}(t) \int_{0}^{t} e^{\int_{1}^{s} p(\tau) d \tau}[q(s, x(s))-\varphi(s)] d s+z^{\prime}(t)\right)^{\prime} \\
= & -h_{1}^{\prime}(t) \int_{0}^{t} e^{\int_{1}^{s} p(\tau) d \tau}[q(s, x(s))-\varphi(s)] d s \\
& -h_{1}(t) e^{\int_{1}^{t} p(\tau) d \tau}[q(t, x(t))-\varphi(t)]+z^{\prime \prime}(t) \\
= & -h_{1}^{\prime}(t) \int_{0}^{t} e^{\int_{1}^{s} p(\tau) d \tau}[q(s, x(s))-\varphi(s)] d s \\
& -[q(t, x(t))-\varphi(t)]+z^{\prime \prime}(t) .
\end{aligned}
$$

It follows from (12) that

$$
\begin{aligned}
x^{\prime \prime}(t)+p(t) x^{\prime}(t)+q(t, x(t)) \\
=-h_{1}^{\prime}(t) \int_{0}^{t} e^{\int_{1}^{s} p(\tau) d \tau}[q(s, x(s))-\varphi(s)] d s \\
\quad-[q(t, x(t))-\varphi(t)]+z^{\prime \prime}(t) \\
\quad-p(t) h_{1}(t) \int_{0}^{t} e^{\int_{1}^{s} p(\tau) d \tau}[q(s, x(s))-\varphi(s)] d s \\
\quad+p(t) z^{\prime}(t)+q(t, x(t)) \\
=z^{\prime \prime}(t)+p(t) z^{\prime}(t)+\varphi(t)=0 .
\end{aligned}
$$

That is, the problem (2) is equivalent to (6). Let us define the recurrence relations

$$
\begin{gathered}
x_{0}(t)=z(t), \\
x_{n}(t)=\int_{0}^{t}\left(E(s) e^{\int_{1}^{s} p(\tau) d \tau}-E(t) e^{\int_{1}^{s} p(\tau) d \tau}\right) \\
\times\left[q\left(s, x_{n-1}(s)\right)-\varphi(s)\right] d s+z(t),
\end{gathered}
$$

where $z(t)$ is a solution of the problem (4). It follows from (9), (10), and (14) that $\alpha<x_{n}(t)<\beta$ for $\alpha<x_{n-1}(t)<\beta$ and for small enough $t \in\left[0, t_{0}\right)$.
Now, for $t_{1}, t_{2} \in\left[0, t_{0}\right)$, we have from (9) and (10) that

$$
\begin{aligned}
\left|x_{n}\left(t_{2}\right)-x_{n}\left(t_{1}\right)\right|=\mid \int_{t_{1}}^{t_{2}}\left(E(s) e^{\int_{1}^{s} p(\tau) d \tau}-E(t) e^{\int_{1}^{s} p(\tau) d \tau}\right) \\
\quad \times\left[q\left(s, x_{n-1}(s)\right)-\varphi(s)\right] d s \mid \\
\leq 2 K\left[\left(t_{2}-\frac{t_{2}^{2}}{2}\right)-\left(t_{1}-\frac{t_{1}^{2}}{2}\right)\right] \\
\leq 2 K\left(t_{2}-t_{1}\right)\left(1+\frac{t_{1}}{2}+\frac{t_{2}}{2}\right) \\
\leq K_{1}\left(t_{2}-t_{1}\right),
\end{aligned}
$$

for some constant $K_{1}$. Thus, the sequence $x_{n}(t)$ is uniformly bounded and uniformly continuous and, by Ascoli-Arzela lemma, there exists a continuous $x(t)$ such that $x_{n_{k}}(t) \rightarrow x(t)$ uniformly on $[0, T]$, for any fixed $T \in\left[0, t_{0}\right)$. Without loss of generality, say $x_{n}(t) \rightarrow x(t)$. Then

$$
\begin{aligned}
x(t)=\lim _{n \rightarrow \infty} \int_{0}^{t}\left(E(s) e^{\int_{1}^{s} p(\tau) d \tau}-E(t) e^{\int_{1}^{s} p(\tau) d \tau}\right) \\
\times\left[q\left(s, x_{n}(s)\right)-\varphi(s)\right] d s+z(t) \\
=\int_{0}^{t}\left(E(s) e^{\int_{1}^{s} p(\tau) d \tau}-E(t) e^{\int_{1}^{s} p(\tau) d \tau}\right) \\
\times[q(s, x(s))-\varphi(s)] d s+z(t),
\end{aligned}
$$

using the Lebesgue dominated convergence theorem.

Note that the positivity condition of the function $p(t)$ can be weakened.

The positivity of $p(t)$ has been used in the proof of Theorem 2 to show the (removable) continuity of the function $h(t)$ at 0 . Now assuming that the following condition holds:

(C2) $|p|$ is integrable on $[c, d]$ for any fixed $c, d \in(0,1]$, $c<d$, and

$$
L \leq \int_{c}^{d} p(s) d s<+\infty ; \quad \text { for some fixed } L
$$

we can prove a similar theorem.

Theorem 3. The conclusion of Theorem 2 remains valid if condition (D2) is replaced by (C2).

Proof. We need to make some modifications to the proof of Theorem 2; for example, instead of the inequality

$$
e^{-\int_{1}^{u} p(v) d v} e^{\int_{1}^{s} p(\tau) d \tau} \leq 1
$$

for $u \geq s$, we will have

$$
e^{-\int_{1}^{u} p(v) d v} e^{\int_{1}^{s} p(\tau) d \tau}=e^{-\int_{s}^{u} p(v) d v} \leq e^{-L},
$$

for small enough $u$ and $s$. 
It is worthy to note that the existence of the solution of the problems like

$$
\begin{array}{r}
x^{\prime \prime}+\left(\frac{a_{m}}{t^{m}}+\frac{a_{m-1}}{t^{m-1}}+\cdots+\frac{a_{1}}{t}+A(t)\right) x^{\prime}+q(t, x(t))=0, \\
x(0)=a, \quad x^{\prime}(0)=b, \quad t>0,
\end{array}
$$

follows from Theorem 2, where $A(t)$ is differentiable function, $q(t, x)$ satisfies the conditions (D4*), $a_{1}, a_{2}, \ldots, a_{m}$ are real constants, and $a_{m}>0$. Indeed for small enough $t$ we have $p(t)>0$ and therefore the hypotheses of Theorems 2 and 3 are true for small enough $t \in[0, T]$; for $b=0$ the problem (4) has a solution $z(t)=a$, and so (21) has a solution for all bounded $q(t, x(t))$ with Caratheodory conditions, but for $b \neq 0$ the problem (21) has a solution for $q(t, x(t))$ with $\left|q(t, x(t))+b\left(a_{m} / t^{m}+a_{m-1} / t^{m-1}+\cdots+a_{1} / t\right)\right|<K$ in some small enough neighborhood of 0 , since the corresponding problem (4) can be taken (e.g.) as

$$
\begin{array}{r}
z^{\prime \prime}+\left(\frac{a_{m}}{t^{m}}+\frac{a_{m-1}}{t^{m-1}}+\cdots+\frac{a_{1}}{t}+A(t)\right) z^{\prime} \\
-b\left(\frac{a_{m}}{t^{m}}+\frac{a_{m-1}}{t^{m-1}}+\cdots+\frac{a_{1}}{t}+A(t)\right)=0, \\
z(0)=a, \quad z^{\prime}(0)=b, \quad t>0,
\end{array}
$$

and has a solution $z(t)=a+b t$. It is remarkable that for $b \neq 0$ the condition for $q(t, x(t))$ can be changed by using different functions for $\varphi(t)$. For example, $\varphi(t)$ can be taken as

$$
\begin{aligned}
\varphi(t)= & \frac{b_{m}}{t^{m}}+\frac{b_{m-2}}{t^{m-2}}+\cdots \\
= & -\frac{b a_{m}}{t^{m}}+\frac{1}{t^{m-2}}\left(\frac{b a_{m-1}^{2}}{a_{m}}-b a_{m-2}\right) \\
& +\frac{1}{t^{m-3}}\left(\frac{b a_{m-1} a_{m-2}}{a_{m}}-b a_{m-3}\right)+\cdots \\
& +\frac{1}{t}\left(\frac{b a_{m-1} a_{2}}{a_{m}}-b a_{1}\right)+\frac{b a_{m-1} a_{1}}{a_{m}}-b A(t)-\frac{b a_{m-1}}{a_{m}}
\end{aligned}
$$

and (4) as

$$
\begin{array}{r}
z^{\prime \prime}+\left(\frac{a_{m}}{t^{m}}+\frac{a_{m-1}}{t^{m-1}}+\cdots+\frac{a_{1}}{t}+A(t)\right) z^{\prime}+\varphi(t)=0, \\
z(0)=a, \quad z^{\prime}(0)=b, \quad t>0,
\end{array}
$$

with solution $z(t)=a+b t-\left(b a_{m-1} / 2 a_{m}\right) t^{2}$. Continuing in like manner, the condition for $q(t, x(t))$ can be reduced to $\left|q(t, x(t))+b a_{m} / t^{m}\right|<K$.

The inequalities of the type (7)-(10) can be easily established for the function $q(t, x)$ with

$$
\left(\mathrm{D} 4^{*} \mathrm{~d}\right)|q(t, x)-\varphi(t)| \leq m(t),
$$

where $m(t)$ is absolutely integrable function, and the more general theorem can be stated as follows.
Theorem 4. The conclusion of Theorem 2 remains valid if the condition $\left(D 4^{*} c\right)$ is replaced by $\left(D 4^{*} d\right)$.

The more applicable version of the existence theorems can be received from Theorems 2, 3, and 4 if the function $\varphi(t)$ is replaced by $\varphi(t, x)$. For example, Theorem 2 can be improved as follows.

Theorem 5. The conclusion of Theorem 2 remains valid if the function $\varphi(t)$ is replaced by $\varphi(t, x)$ and $(4)$ is replaced by

$$
x^{\prime \prime}+p(t) x^{\prime}+\varphi(t, x(t))=0, \quad x(0)=a, t>0,
$$

where $\varphi(t, x(t))$ is a function with Caratheodory conditions $(D 4 * a)$ and $(D 4 * b)$.

The "traditional" uniqueness theorems when $q(t, x)$ is Lipschitz in $x$ on $[\alpha, \beta]$ can also be established.

Theorem 6. Suppose the conditions of Theorem 2 or Theorem 3 hold and, in addition, suppose that $q$ is Lipschitz in $x$ on $[\alpha, \beta]$. Then the IVP (2) has a unique solution.

Proof (see also [19]). Suppose $x_{1}(t), x_{2}(t)$ are solutions to (2) on $[0, T]$ for some $T \in(0,1]$. Since $q$ is Lipschitz in $x$ on $[\alpha, \beta]$, there exists $L>0$ such that $\left|q\left(t, x_{1}\right)-q\left(t, x_{2}\right)\right| \leq L\left|x_{1}-x_{2}\right|$, whenever $x_{1}, x_{2} \in[\alpha, \beta]$ and $t \in[0,1]$. From (6) it follows that, for $t \in[0, T]$,

$$
\begin{array}{r}
x_{i}(t)=\int_{0}^{t}\left(E(s) e^{\int_{1}^{s} p(\tau) d \tau}-E(t) e^{\int_{1}^{s} p(\tau) d \tau}\right) \\
\times\left[q\left(s, x_{i}(s)\right)-\varphi(s)\right] d s+z(t), \\
i=1,2,
\end{array}
$$

and so

$$
\begin{aligned}
\left|x_{2}(t)-x_{1}(t)\right|= & \mid \int_{0}^{t}\left(E(s) e^{\int_{1}^{s} p(\tau) d \tau}-E(t) e^{\int_{1}^{s} p(\tau) d \tau}\right) \\
& \times\left[q\left(s, x_{2}(s)\right)-q\left(s, x_{1}(s)\right)\right] d s \mid \\
& \leq K_{3} \int_{0}^{t}\left|x_{2}(s)-x_{1}(s)\right| d s
\end{aligned}
$$

for some constant $K_{3}$ (see inequalities (9) and (10)).

Now we use Gronwall's lemma (see, e.g., [21]). Applying this lemma with $\sigma=0$ and $r(s)=\left|x_{2}(s)-x_{1}(s)\right|$ yields $\mid x_{2}(t)-$ $x_{1}(t) \mid \leq 0$, from which it follows that $x_{2}(t)=x_{1}(t)$, thereby proving the theorem.

Remark 7. Biles et al. [19] give an example which satisfies conditions of Theorem 1 except condition $p \geq 0$. They considered the problem

$$
x^{\prime \prime}-t^{-1} x^{\prime}=0, \quad x(0)=1, x^{\prime}(0)=0,
$$

with the family of solutions $x=c t^{2}+1$, where $c$ is an arbitrary constant. 
Note that here $S \equiv\left\{\int_{a}^{b} p(s) d s: a \leq b \in(0,1)\right\}=(-\infty, 0]$. Thus, in fact, not the condition $p(s) \geq 0$ but the boundedness below of the set $S$ is important for the uniqueness.

\section{Applications}

Now we can find wide classes of IVPs with corresponding existence and uniqueness criteria. The class of solvable problems can be extended by adding a function $\varphi(t)$ to the function $q(t, x)$, where $\varphi(t)$ is taken from the equation of the type (4) with a solution.

Let us rephrase the main conclusion of Theorem 5 as follows. If the (singular) problem

$$
\begin{aligned}
& x^{\prime \prime}+p(t) x^{\prime}+\varphi(t, x(t))=0, \\
& x(0)=a, \quad x^{\prime}(0)=b, \quad t>0,
\end{aligned}
$$

has a solution, then the problem

$$
\begin{array}{r}
x^{\prime \prime}+p(t) x^{\prime}+\varphi(t, x(t))+q(t, x(t))=0, \\
x(0)=a, \quad x^{\prime}(0)=b, \quad t>0,
\end{array}
$$

where $q(t, x)$ is a bounded function with Caratheodory conditions, has a solution as well.

Example 8. The problem

$$
\begin{array}{r}
x^{\prime \prime}+p(t) x^{\prime}+q(t, x(t))-b p(t)=0, \\
x(0)=a, \quad x^{\prime}(0)=b, \quad t \geq 0,
\end{array}
$$

has a solution for all bounded $q(t, x(t))$. Indeed the problem

$$
z^{\prime \prime}(t)+p(t) z^{\prime}(t)-b p(t)=0, \quad z(0)=a, \quad z^{\prime}(0)=b,
$$

has a solution $z(t)=b t+a$. Then existence of solution of (32) follows from Theorem 2 .

Example 9. Consider the problem

$$
\begin{aligned}
& x^{\prime \prime}+\frac{k}{t^{r}} x^{\prime}+c t^{m} f(x)-\frac{b k}{t^{r}}=0, \\
& x(0)=a, \quad x^{\prime}(0)=b, \quad t \geq 0,
\end{aligned}
$$

where $k \geq 0, r \in(0, \infty), m>-1$, and $f(x)$ is bounded function. It follows from Theorem 4 that this problem has a solution. The functions $m(t)$ and $\varphi(t)$ can be taken as (const) $t^{m}$ and $-b k / t^{r}$, respectively. The equation corresponding to (4)

$$
z^{\prime \prime}+\frac{k}{t^{r}} z^{\prime}-\frac{b k}{t^{r}}=0, \quad z(0)=a, \quad z^{\prime}(0)=b, \quad t \geq 0,
$$

has a solution $z(t)=a+b t$. The case $b=0$ and $f(x)=x^{n}$ correspond to the standard Emden-Fowler equation.
Example 10. Now consider the problem

$$
x^{\prime \prime}+\frac{k}{t^{r}} x^{\prime}+\left(\ln ^{n} t\right) t^{m} g(x)=0, \quad x(0)=a, \quad x^{\prime}(0)=0,
$$

where $g(x)$ is continuous on $[0,1]$ function, $k \geq 0, r \in$ $(-\infty, \infty), n \geq 0$, and $m>-1$.

Since

$$
t^{\alpha} \ln ^{n} t \longrightarrow 0 \quad \text { as } t \longrightarrow 0,
$$

for any $\alpha>0$ and $r \geq 0$, we have that $\left(\ln ^{n} t\right) t^{m}$ is integrable and so the problem has a solution. For the approximate solution of the problems like (36) see [22].

Example 11. The problem

$$
\begin{array}{r}
x^{\prime \prime}+x^{\prime} \sin \frac{1}{t}-\frac{x}{t} \sin \frac{1}{t}+f(t) g(x)=0, \\
x(0)=0, \quad x^{\prime}(0)=1, \quad t \geq 0,
\end{array}
$$

where $f(t), g(x)$ are continuous functions, satisfies the conditions of Theorem 3. Indeed, the problem

$$
\begin{aligned}
& x^{\prime \prime}+x^{\prime} \sin \frac{1}{t}-\frac{x}{t} \sin \frac{1}{t}=0, \\
& x(0)=0, \quad x^{\prime}(0)=1, \quad t \geq 0,
\end{aligned}
$$

has a solution $x(t)=t$. It is worthy to note that every neighborhood of 0 contains the points $t_{1}, t_{2}$ with $p\left(t_{1}\right)>0$ and $p\left(t_{2}\right)<0$.

\section{Concluding Remarks}

We extended the class of solvable second-order singular IVPs. We established that the difficulties related to the singularity can be overcome for the problems of the type (2) with $p \geq 0$ or

$$
L \leq \int_{c}^{d} p(s) d s<+\infty ; \quad \text { for some fixed } L .
$$

The problem of the existence of a solution is reduced to the finding of a solution of some more easy problems like (4).

The approach used here can be useful for the problems on the existence of solutions of boundary value problems [23$26]$. The authors in $[23,24]$ established remarkable theorems on the existence and uniqueness of the solution of the equation

$$
u^{\prime \prime}+p(t) u^{\prime}+q(t) u+\varphi(t)=0,
$$

with some boundary conditions, in terms of an auxiliary homogeneous equation

$$
u^{\prime \prime}+p(t) u^{\prime}+q(t) u=0 .
$$


Our approach is different from the approach in [23-25]. We consider the new auxiliary (nonhomogeneous, but easily solvable) (4) instead of (42).

The conditions we obtained are weaker than the previously known ones and can be easily reduced to several special cases.

\section{Conflict of Interests}

The author declares that there is no conflict of interests regarding the publication of this paper.

\section{References}

[1] M. Beech, "An approximate solution for the polytrope $\mathrm{n}=3$," Astrophysics and Space Science, vol. 132, no. 2, pp. 393-396, 1987.

[2] S. Chandrasekhar, Introduction To the Study of Stellar Structure, Dover, New York, NY, USA, 1967.

[3] E. A. Coddington and N. Levinson, Theory of Ordinary Differential Equations, Tata McGraw-Hill, Bombay, India, 1955.

[4] G. P. Horedt, "Exact solutions of the Lane-Emden equation in $N$-dimensional space," Astronomy and Astrophysics, vol. 172, no. 1, pp. 359-367, 1987.

[5] S. Liao, "A new analytic algorithm of Lane-Emden type equations," Applied Mathematics and Computation, vol. 142, no. 1, pp. 1-16, 2003.

[6] F. K. Liu, "Polytropic gas spheres: an approximate analytic solution of the Lane-Emden equation," Monthly Notices of the Royal Astronomical Society, vol. 281, pp. 1197-1205, 1996.

[7] Z. F. Seidov and R. K. Kyzakhmetov, "Solutions of the LaneEmden problem in series," Soviet Astronomy, vol. 21, pp. 399400, 1977.

[8] N. T. Shawagfeh, "Nonperturbative approximate solution for Lane-Emden equation," Journal of Mathematical Physics, vol. 34, no. 9, pp. 4364-4369, 1993.

[9] H. T. Davis, Introduction to Nonlinear Differential and Integral Equations, Dover, New York, NY, USA, 1962.

[10] P. Rosenau, "A note on integration of the Emden-Fowler equation," International Journal of Non-Linear Mechanics, vol. 19, no. 4, pp. 303-308, 1984.

[11] R. Bellman, Stability Theory of Differential Equations, McGrawHill, New York, NY, USA, 1953.

[12] P. Habets and F. Zanolin, "Upper and lower solutions for a generalized Emden-Fowler equation," Journal of Mathematical Analysis and Applications, vol. 181, no. 3, pp. 684-700, 1994.

[13] D. Hinton, "An oscillation criterion for solutions of $\left(r y^{\prime}\right)^{2}+$ $q y^{\gamma}=0$," The Michigan Mathematical Journal, vol. 16, pp. 349$352,1969$.

[14] J. Janus and J. Myjak, "A generalized Emden-Fowler equation with a negative exponent," Nonlinear Analysis. Theory, Methods \& Applications, vol. 23, no. 8, pp. 953-970, 1994.

[15] P. G. L. Leach, "First integrals for the modified Emden equation $q^{\prime \prime}+\alpha(t) q^{\prime}+q^{n}=0$," Journal of Mathematical Physics, vol. 26, no. 10, pp. 2510-2514, 1985.

[16] W. Mydlarczyk, "A singular initial value problem for second and third order differential equations," Colloquium Mathematicum, vol. 68 , no. 2, pp. 249-257, 1995.

[17] O. U. Richardson, The Emission of Electricity From Hot Bodies, Longman's Green and Company, London, UK, 1921.
[18] A.-M. Wazwaz, "A new algorithm for solving differential equations of Lane-Emden type," Applied Mathematics and Computation, vol. 118, no. 2-3, pp. 287-310, 2001.

[19] D. C. Biles, M. P. Robinson, and J. S. Spraker, "A generalization of the Lane-Emden equation," Journal of Mathematical Analysis and Applications, vol. 273, no. 2, pp. 654-666, 2002.

[20] R. P. Agarwal and D. O'Regan, "Second order initial value problems of Lane-Emden type," Applied Mathematics Letters, vol. 20, no. 12, pp. 1198-1205, 2007.

[21] R. K. Miller and A. N. Michel, Ordinary Differential Equations, Academic Press, New York, NY, USA, 1982.

[22] A. Aslanov, "A generalization of the Lane-Emden equation," International Journal of Computer Mathematics, vol. 85, no. 11, pp. 1709-1725, 2008.

[23] I. T. Kiguradze and A. G. Lomtatidze, "On certain boundary value problems for second-order linear ordinary differential equations with singularities," Journal of Mathematical Analysis and Applications, vol. 101, no. 2, pp. 325-347, 1984.

[24] A. Lomtatidze, "On a nonlocal boundary value problem for second order linear ordinary differential equations," Journal of Mathematical Analysis and Applications, vol. 193, no. 3, pp. 889908, 1995.

[25] A. Lomtatidze and L. Malaguti, "On a two-point boundary value problem for the second order ordinary differential equations with singularities," Nonlinear Analysis, Theory, Methods and Applications, vol. 52, no. 6, pp. 1553-1567, 2003.

[26] R. P. Agarwal and I. Kiguradze, "On multi-point boundary value problems for linear ordinary differential equations with singularities," Journal of Mathematical Analysis and Applications, vol. 297, no. 1, pp. 131-151, 2004. 


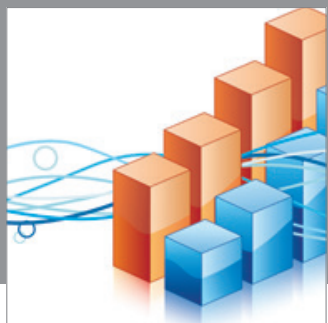

Advances in

Operations Research

mansans

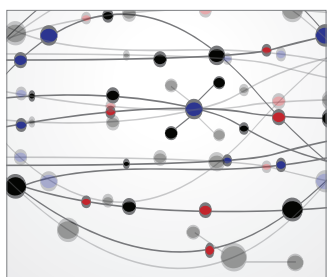

The Scientific World Journal
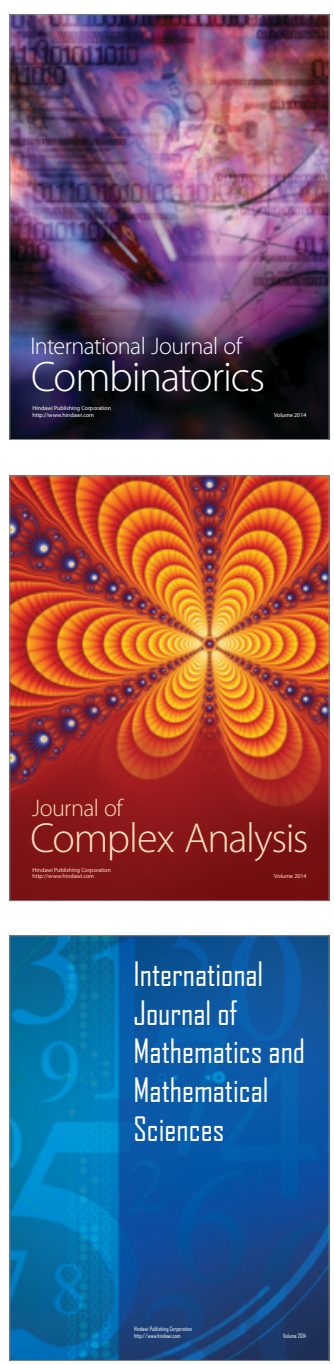
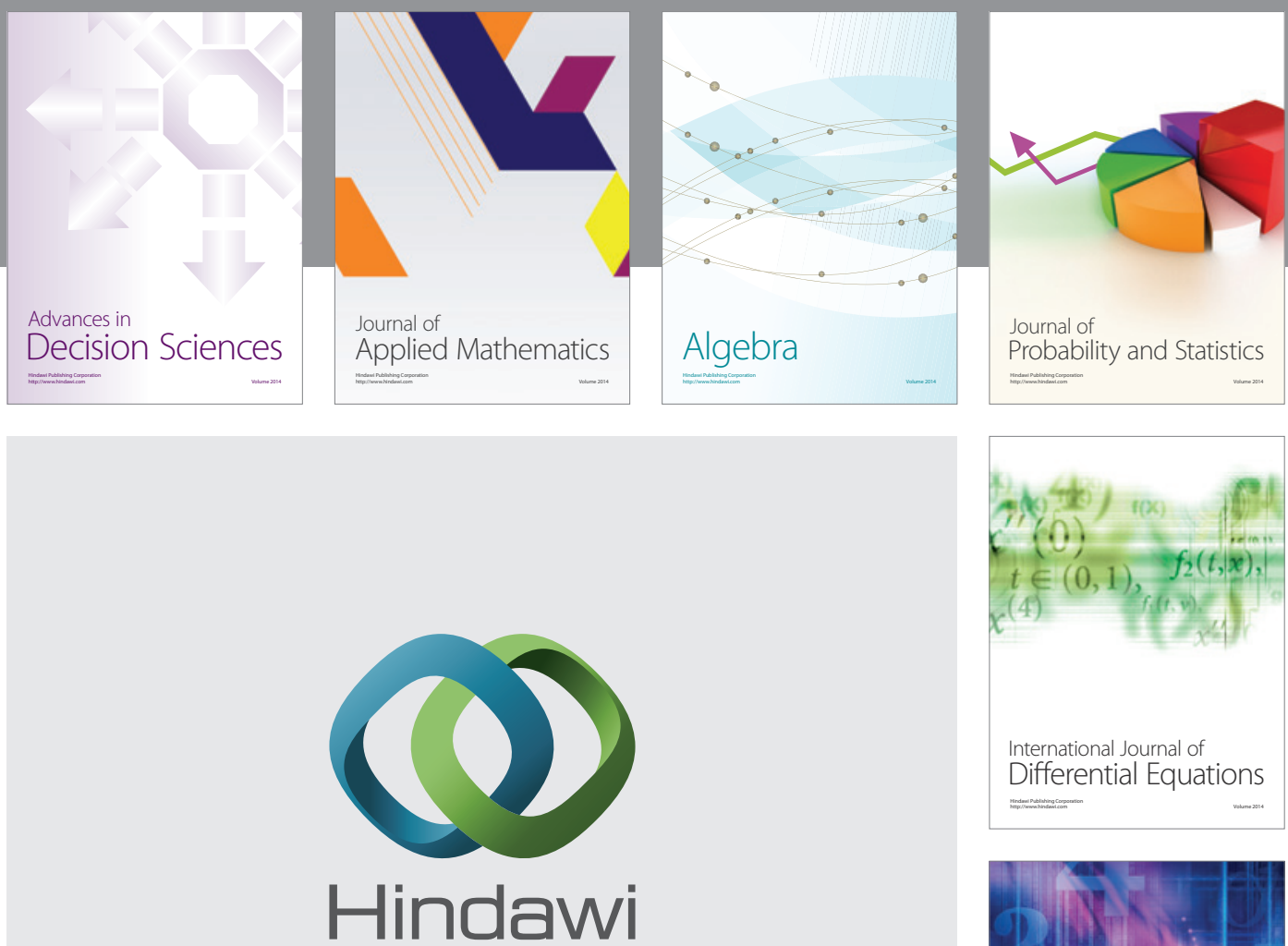

Submit your manuscripts at http://www.hindawi.com
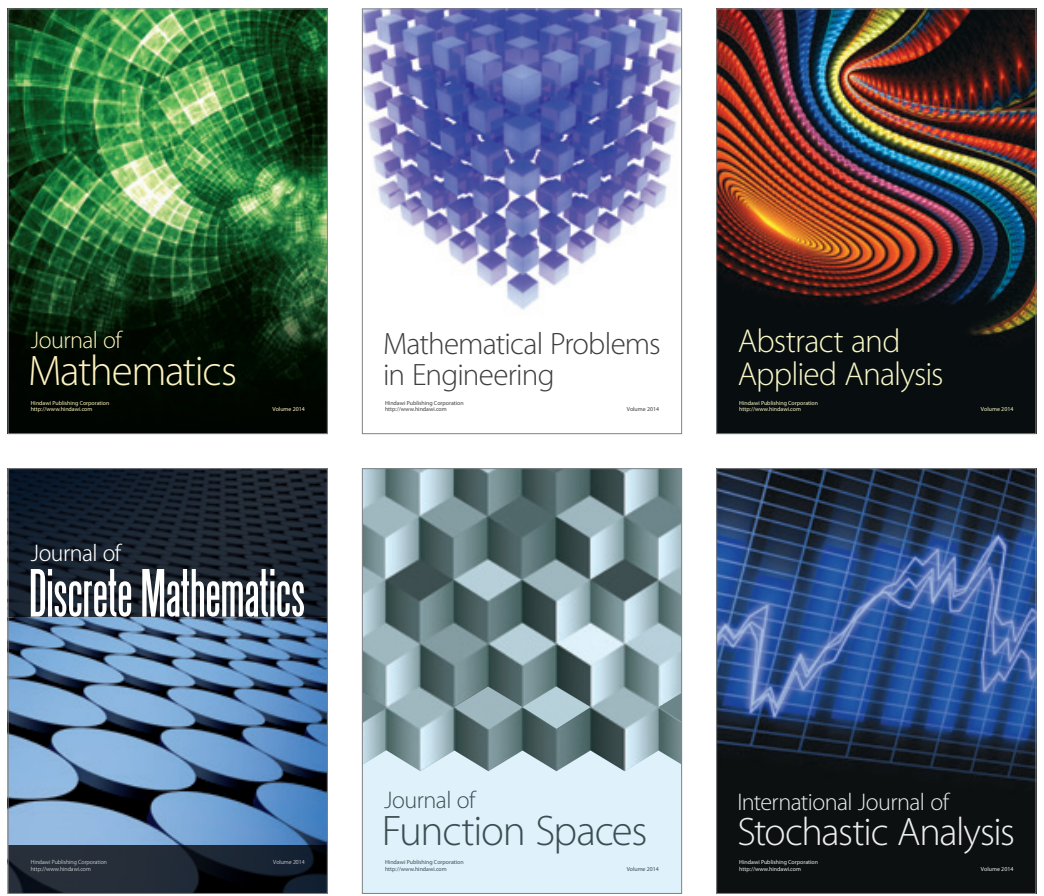

Journal of

Function Spaces

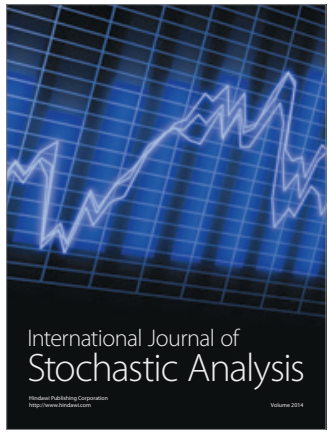

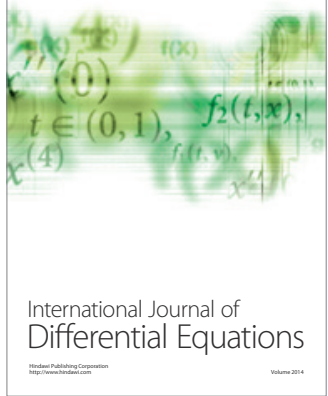
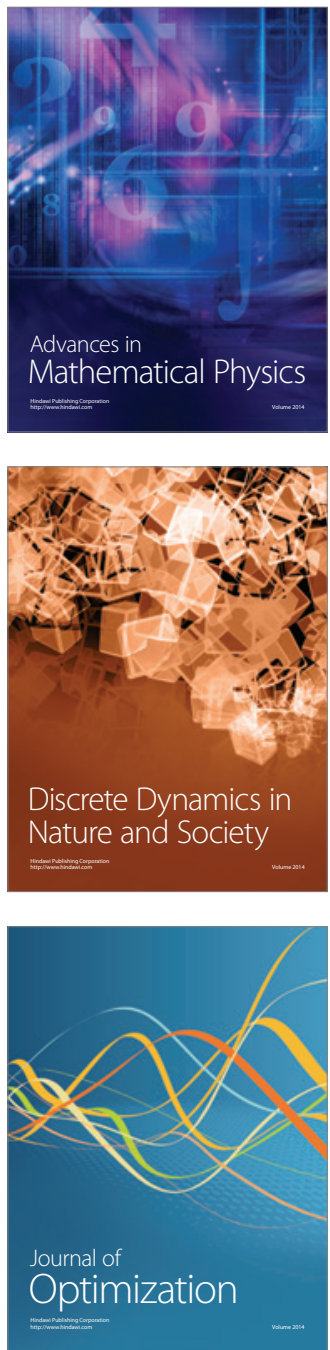